\title{
Hypoxic-ischaemic encephalopathy: early and late magnetic resonance imaging findings in relation to outcome
}

\author{
Mary Rutherford, Jacqueline Pennock, Jane Schwieso, Frances Cowan, Lilly Dubowitz
}

\begin{abstract}
Sixteen infants with hypoxic-ischaemic encephalopathy (HIE) were studied using serial magnetic resonance imaging (MRI) up to the age of 2 years. The infants had regular neurological and developmental assessments. An nuclear magnetic resonance (NMR) score was devised to quantify the early and late MRI findings and a neurological optimality score was used to quantify abnormal neurological signs at the time of the final examination. The follow up MRI score was compared with the neonatal MRI score and the outcome of the child.
\end{abstract}

There was a strong positive correlation between the neonatal and follow up MRI scores and between MRI scores and optimality score. All infants with a normal outcome had patchy white matter abnormalities. All infants with an abnormal outcome had extensive white matter abnormalities. The outcome was most severe in those infants with additional basal ganglia atrophy with or without cyst formation.

Infants with mild HIE who are developmentally normal at the age of 2 years do not have normal MRI scans and may be at risk of minor neurological problems by school age. Bilateral basal ganglia abnormalities are associated with severe developmental delay, but infants with mainly white matter and cortical abnormalities have less severe problems despite extensive tissue loss.

Keywords: hypoxic-ischaemic encephalopathy, magnetic resonance imaging, optimality score, signal intensity.

Department of Paediatrics and Neonatal Medicine, Hammersmith Hospital,

Du Cane Road,

London W12 OHS

M Rutherford

F Cowan

L Dubowitz

Robert Steiner MRI Unit,

Hammersmith

Hospital

Jacqueline Pennock

Jane Schwieso

Correspondence to: Dr Mary Rutherford.

Accepted

10 September 1996 capsule are associated with structural changes in the brain at 3 months of age, ${ }^{10}$ but this is too early to assess clinical outcome. Magnetic resonance images continue to change in appearance because of normal development, compensatory mechanisms, and secondary degeneration as a result of the initial injury. There are few studies that document both neonatal and late MRI findings in infants with HIE and detailed clinical outcomes are not given. ${ }^{19}$

The aim of this study was to document the MRI findings in infants with HIE between 12 and 24 months of age and relate these to: (i) magnetic resonance imaging in the neonatal period; and (ii) the clinical outcome at the time of the final scan.

\section{Methods}

Infants were included in the study if they met all the following criteria: (i) term or post-term (37-43 weeks'gestation); (ii) evidence of fetal distress; (iii) neurologically abnormal in the first 48 hours of life, with abnormalities of tone with or without convulsions and altered consciousness. Fetal distress was diagnosed in the presence of cardiotocographic abnormalities of bradycardia $(<100 /$ minute or late decelarations (type II dips) with or without meconium stained liquor and with low Apgar scores and the necessity for resuscitation. Hypoxic-ischaemic encephalopathy was classified as mild, moderate, or severe (I, II, or III) according to Sarnat and Sarnat. ${ }^{11}$

All infants had at least one MRI scan performed within the first 4 weeks of life and one scan between 12 and 24 months of age.

MAGNETIC RESONANCE IMAGING

Magnetic resonance imaging was performed using a Picker HPQ Vista system (Cleveland, Ohio) operating at 1.0 Tesla. $T_{1}$ and $T_{2}$ weighted spin echo $\left(T_{1}\right.$ WSE and $T_{2}$ WSE) sequences and age related inversion recovery (IR) sequences were used. In some infants a fluid attenuated inversion recovery (FLAIR) sequence was also used. ${ }^{12}$ The detailed scanning variables are given in table 1 .

The MRI studies were carried out with the infant's head placed laterally in a specifically designed $24 \mathrm{~cm}$ split, clear receiver coil. Images were obtained in the transverse plane. Immobilisation was achieved by surrounding the head with an air evacuated bag filled with polystyrene balls. Neonates were examined unsedated during natural sleep. Older infants were sedated with chloral hydrate $60-100 \mathrm{mg} / \mathrm{kg}$ orally, or rectally as a suppository. All children 
Table 1 Parameters for MRI sequences

\begin{tabular}{lccc}
\hline & Repetition time (ms) & Echo time (ms) & Inversion time (ms) \\
\hline Inversion recovery & $3600-3800$ & 30 & $700-950$ \\
FLAIR & 6500 & 160 & 2100 \\
$\mathrm{~T}_{1}$ weighted spin echo & 860 & 20 & \\
$\mathrm{~T}_{2}$ weighted spin echo & 3400 & 120 & \\
\hline
\end{tabular}

were monitored with ECG and pulse oximetry. A paediatrician was present throughout the investigation.

Abnormal SI within the basal ganglia and thalami may predict outcome and a separate detailed score for the basal ganglia and thalami (TBG) was used, as described before. ${ }^{8}$ The maximum score was 18 .

The neonatal NMR score was obtained from first week scans. ${ }^{10}$ Points were given for the presence of brain swelling and abnormal signal intensities within the cortex, subcortical and periventricular white matter, basal ganglia and thalami (less detailed than in the TBG score) and from the posterior limb of the internal capsule. The maximum score was 17 .

The follow up NMR score was adapted from the neonatal scoring system ${ }^{10}$ and included points for atrophy and delayed myelination, but not for brain swelling as this was not seen after the first week of life. The IR sequence and the $T_{2}$ WSE sequence images were assessed visually and a score reached by consensus. A comparison was made with scans of neurologically normal infants of equivalent age. Details of the follow up score are shown in table 2.

The late MRI findings were compared with the neonatal MRI findings and with the clinical outcome of the infant.

FOLLOW UP AND CLINICAL OUTCOME

Infants had a detailed neurological examination ${ }^{13}$ and a Griffiths developmental assessment at three monthly intervals over the first two years of life. All infants were over 1 year of age at their final assessment. Clinical outcome was classified as normal or abnormal. Those infants with an abnormal outcome were divided into groups according to the severity of their developmental delay.

Normal outcome-A normal neurological and developmental assessment, although there may have been transient disturbances of tone during the first year of life.

Abnormal outcome-These infants had persistent neurological signs consistent with a central motor deficit, with or without developmental delay.

(a) No developmental delay

(b) Moderate developmental delay

(c) Severe developmental delay

(d) Very severe developmental delay (no discernible development)

The central motor deficit was classified as a hemiplegia, spastic quadriplegia, athetoid quadriplegia or a mixed quadriplegia (spastic with athetoid/dystonic movements).

\section{OPTIMALITY SCORE}

A neurological optimality score was given to each child at their last examination to give a quantifiable assessment of their neurological function. This was adapted from a proforma used by Kuenzle. ${ }^{7}$ The optimality score combined both developmental and neurological findings and was easy to complete even in severely abnormal infants. The proforma scored the following areas separately: tone, posture, spontaneous motility, elicited motility, interaction and reflexes. A mean score was given in each area and a total mean score gave the optimality score. The maximum optimality score and therefore a normal result was 21 .

\section{Results}

The 16 infants had a total of 95 follow up scans, 36 after the age of 6 months. All infants had at least one scan between 1 and 2 years of age. The abnormal imaging findings are given in table 3 and the MRI scores are given in table 4 .

All sixteen infants had abnormalities in the white matter on follow up scans. In eight of these infants the changes were mild and patchy and limited to the periventricular white matter (fig 1). One of these eight infants had mild ventricular dilatation, but in the remaining seven the scans were otherwise normal. All eight infants had had signs of brain swelling on their first week scan, but only one had a persistent area of abnormal SI in the white matter (case7).

Table 2 Clinical neonatal details and optimality scores

\begin{tabular}{llllllll}
\hline Case No & Gestation & Fetal distress & $\begin{array}{l}\text { Apgar score } 1,5 \\
\text { minutes }\end{array}$ & Delivery & HIE grade & Outcome & $\begin{array}{l}\text { Optimality } \\
\text { score }\end{array}$ \\
\hline 1 & 40 & ctg/msl & 3,7 & Ventouse & II & Normal & 21 \\
2 & 40 & nil & 4,4 & nvd & I & Normal & 20.7 \\
3 & 41 & ctg/tachy & 4,4 & nvd & III & Normal & 20.5 \\
4 & 40 & ctg & 2,7 & nvd & I & Normal & 21 \\
5 & 41 & NR & 3,4 & nvd & I & Normal & 21 \\
6 & 41 & ctg/msl & 2,6 & Ventouse & I & Normal & 20.9 \\
7 & 43 & NR & 0,0 & nvd & II & Hemisyndrome & 20.3 \\
8 & 39 & ctg/msl & 2,3 & nvd & II & Hemiplegia & 20.3 \\
9 & 40 & ctg & 1,2 & emcs & II & cmd/mdd & 16.4 \\
10 & 38 & ctg & 2,5 & emcs & II & cmd/sdd & 14.6 \\
11 & 40 & PA & 2,6 & emcs & II & cmd/sdd & 11.9 \\
12 & 41 & ctg & 0,0 & Ventouse & II & cmd/sdd & 11.7 \\
13 & 42 & ctg & 0,0 & Forceps & II & cmd/sdd & 9.0 \\
14 & 41 & ctg/msl & 1, & Ventouse & II & cmd/sdd & 8.8 \\
15 & 41 & ctg & 2,5 & emcs & II & cmd/vsdd & 7.6 \\
16 & 41 & ctg/msl & 0,4 & emcs & III & cmd/vsdd & 6.3
\end{tabular}

CTG = cardiotocograph; $\mathrm{msl}=$ meconium stained liquor; emcs = emergency caesarean section; NR = not recorded; PA = placental abruption; $\mathrm{cmd}$ = central motor deficit; mdd = moderate developmental delay; sdd = severe developmental delay; vsdd = very severe developmental delay. 


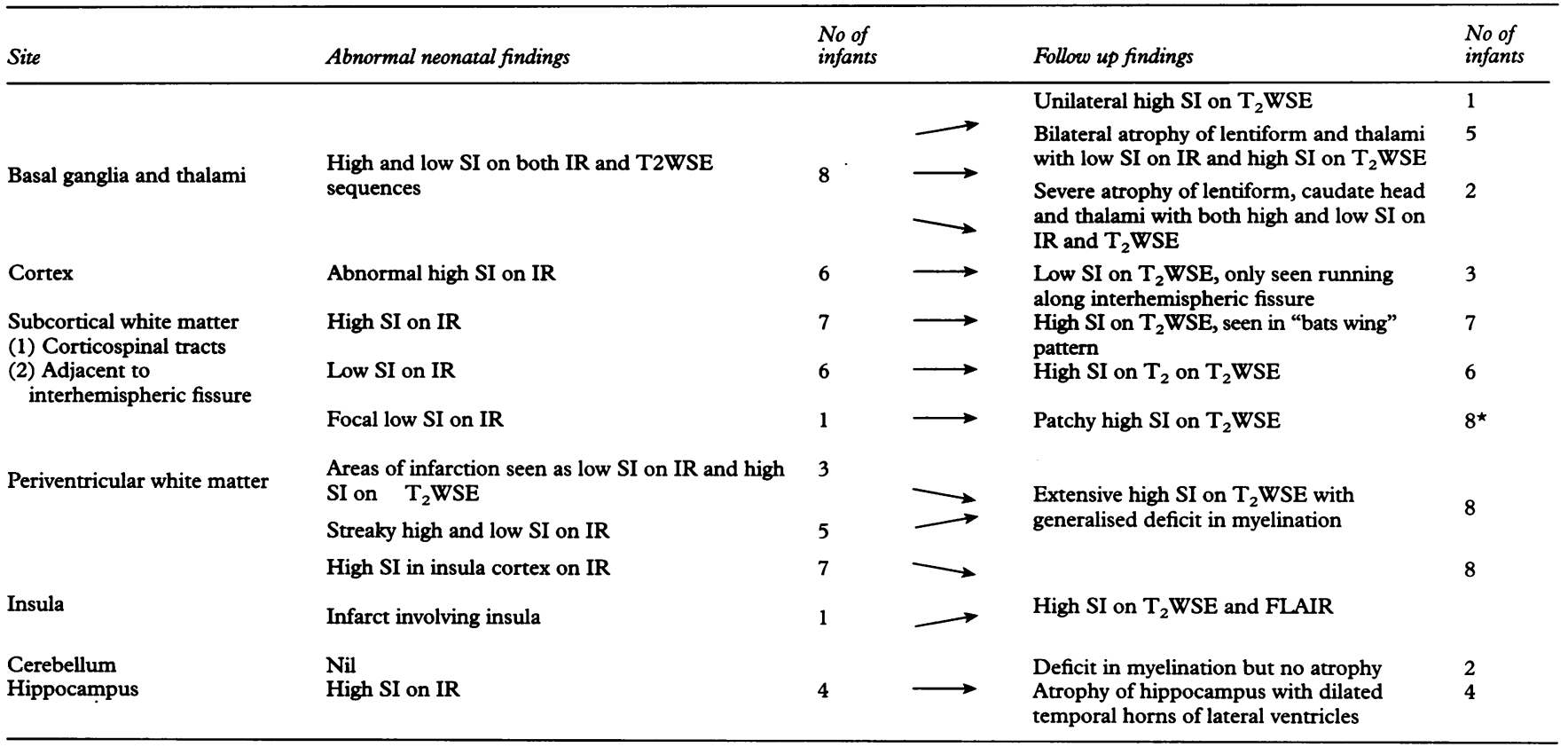

^ All infants had signs of brain swelling on neonatal scan.

Table 4 MRI and optimality scores

\begin{tabular}{|c|c|c|c|c|c|}
\hline \multirow[b]{2}{*}{ Case No } & \multicolumn{2}{|c|}{ Neonatal scan score } & \multicolumn{2}{|c|}{ Follow up scan score } & \multirow{2}{*}{$\begin{array}{l}\text { Optimality } \\
\text { score }\end{array}$} \\
\hline & $T B G(\max =18)$ & Total $(\max =17)$ & $T B G(\max =18)$ & Total $(\max =46)$ & \\
\hline 1 & 0 & 3 & 0 & 4 & 21 \\
\hline 2 & 0 & 0 & 0 & 2 & 20.7 \\
\hline 3 & 2 & 3 & 0 & 2 & 20.5 \\
\hline 4 & 0 & 2 & 0 & 4 & 21 \\
\hline 5 & 2 & 4 & 0 & 5 & 21 \\
\hline 6 & 2 & 3 & 0 & 2 & 20.9 \\
\hline 7 & 0 & 2 & 0 & 3 & 20.3 \\
\hline 8 & 0 & 3 & 0 & 4 & 20.3 \\
\hline 9 & 3 & $7 \star$ & 2 & 16 & 16.4 \\
\hline 10 & 12 & 14 & 10 & 31 & 14.6 \\
\hline 11 & 12 & 11 & 10 & 28 & 11.9 \\
\hline 12 & 15 & $11^{\star}$ & 6 & 20 & 11.7 \\
\hline 13 & 12 & 13 & 16 & 37 & 9.0 \\
\hline 14 & 12 & $9 \star$ & 14 & 28 & 8.1 \\
\hline 15 & 18 & 12 & 18 & 46 & 7.6 \\
\hline 16 & 18 & 13 & 18 & 45 & 6.3 \\
\hline
\end{tabular}

^ Minimum score.

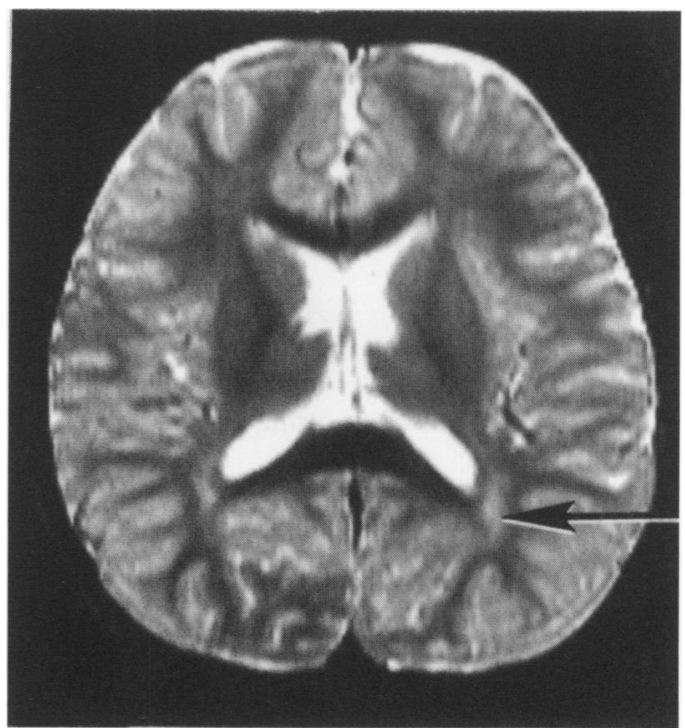

Figure 1 Boy with grade I HIE aged 1 year (case 5). $T_{2}$ weighted spin echo sequence (SE 3000/120). Minor bilateral SI is increased in posterior periventricular white matter at 1 year of age (arrow).

Hippocampal atrophy was only seen in infants with severe basal ganglia abnormalities.

There was a positive correlation between the neonatal and follow up TBG scores (Kendall's rank correlation, $\mathrm{P}=0.0005)$. There was a
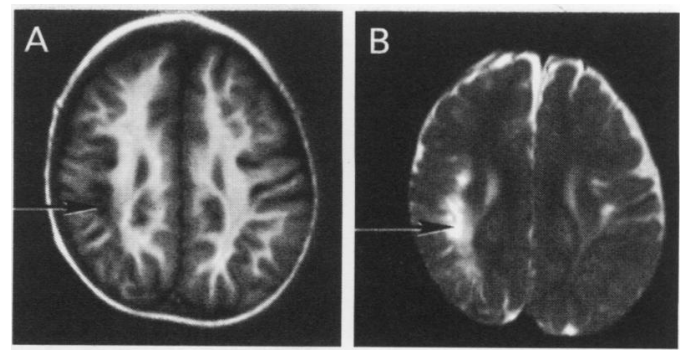

Figures $2 A$ and B Boy with grade II HIE (case 12).

Inversion recovery sequence (IR 3600/30/800) $(A)$ and $T_{2}$ weighted spin echo sequence (SE 3000/120) (B). Generalised myelination deficit is evident $(A)$ with increased $T_{2}$ most evident in the region of a parietal infarct (arrows) (B). 

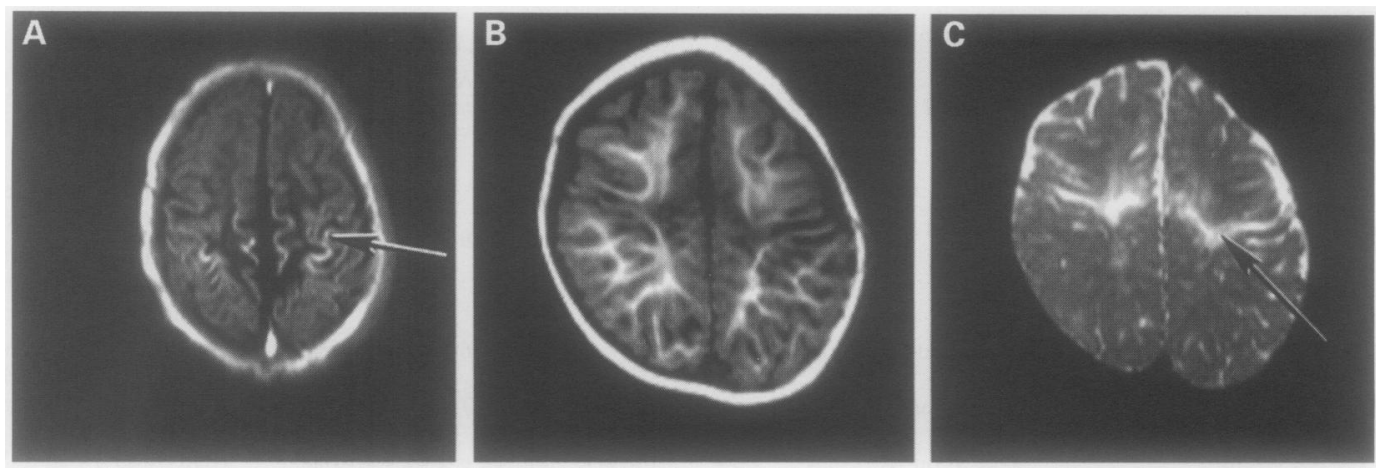

Figures $3 A$ and B Girl with grade II HIE (case 15). Inversion recovery sequence (IR 3800/30/950) (A), inversion recovery sequence (IR 3600/30/800) (B), and $T_{2}$ weighted spin echo sequence (SE 3400/120) (C). There is abnormal increased signal intensity running along the corticospinal tracts in the centrum semiovale adjacent to the Rolandic fissure on the three week scan (arrow) $(A)$ and at 15 months increased $T_{2}$ (arrow) (C). There is a generalised myelination deficit with some widening of the interhemispheric fissure (B).

strong positive correlation between the neonatal and follow up NMR scores (Kendall's rank correlation, $\mathrm{P}=0.0001$ ).

NEONATAL AND FOLLOW UP MRI FINDINGS IN RELATION TO CLINICAL OUTCOME

Normal outcome $(n=6)$ :

Six children had a normal outcome (optimality scores 20.5.-21).These children had patchy white matter abnormalities on follow up scan (NMR score 1-2). Five infants had mild brain swelling on the neonatal scan, and two of these also had minor SI changes in the lentiform nuclei. The remaining infant had a subarachnoid haemorrhage.

Abnormal outcome $(n=10)$ :

Eight infants had a central motor deficit involving all four limbs and two infants had unilateral signs.

Normal development $(n=2)$ :

Two infants had minor neurological signs and normal development (optimality scores were 20.3). One infant had a mild hemiplegia and one a mild hemisyndrome. The $\mathrm{T}_{2} \mathrm{WSE}$ follow up scans showed patchy increased SI in the periventricular white matter in both infants (NMR scores 2, 3). There were no focal lesions or evidence of brainstem asymmetry.

Both infants had brain swelling on the neonatal scans and the infant with the
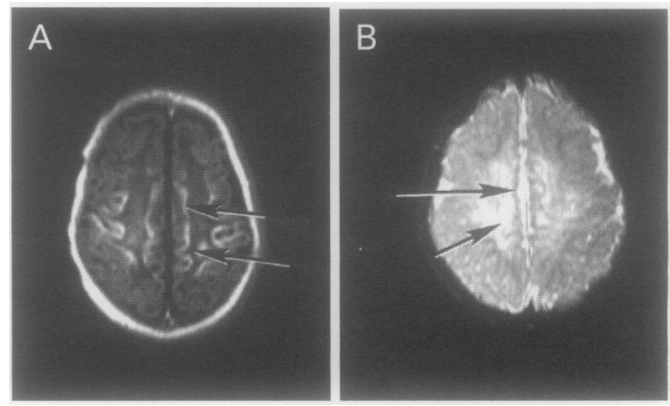

Figures $4 A$ and $B$ Girl with grade II HIE (case 9).

Inversion recovery sequence (IR 3800/30/950) $(A)$ and $T_{2}$ weighted spin echo sequence (SE 3000/120). There is bilateral highlighting of the cortex (short arrow), with low signal areas in the adjacent subcortical white matter long arrow) at 3 weeks of age (A). At 15 months bilateral low signal is seen in the cortex running parallel to the interhemispheric fissure (long arrow) There is bilateral high signal in the subcortical white matter (short arrow) (B).

hemisyndrome also had persistent low signal on inversion recovery sequence in the right posterior parietal white matter.

Moderate developmental delay $(n=1)$ :

One infant had a central motor deficit with moderate developmental delay (optimality score 16.4). The signs consisted of secondary microcephaly, extensor posturing and increased tone in lower limbs. In spite of this at the age of 2 years she was able to cruise around the furniture. She had a vocabulary of several words. Follow up scans showed pronounced
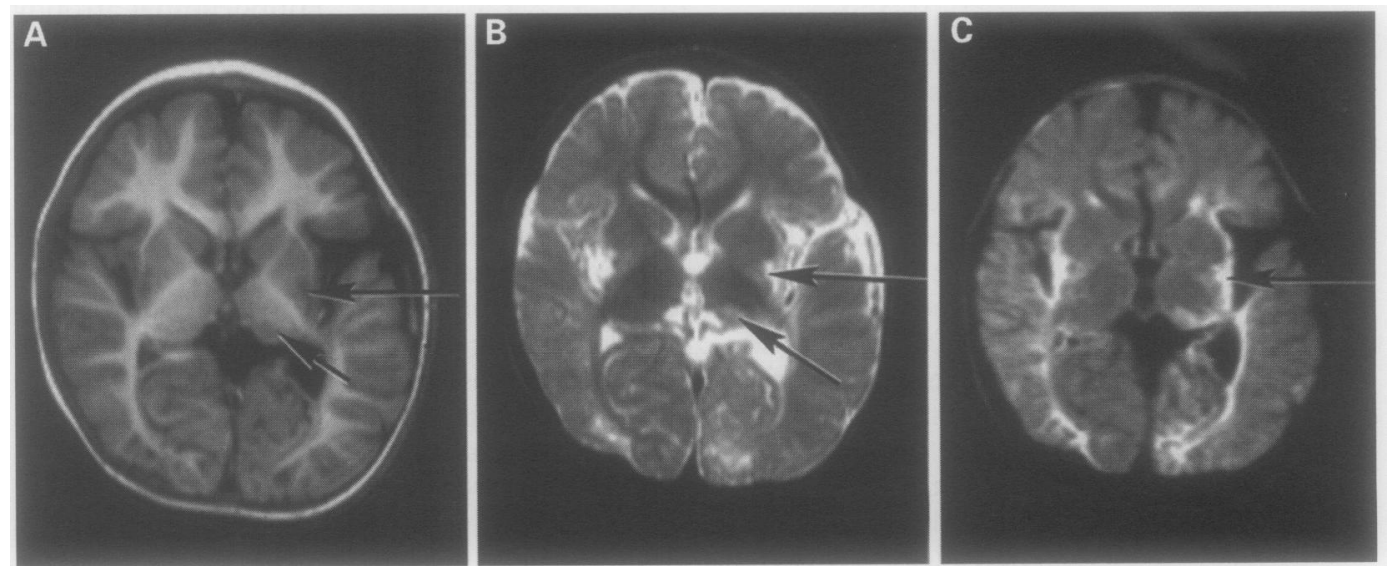

Figures 5A, B, and C Boy with grade II HIE aged 1 year (case 12). Inversion recovery sequence (IR 3600/30/800) (A), $T$ weighted spin echo sequence (SE 3000/ 120) (B), and FLAIR (fluid attenuated IR 6500/160/2100) (C). There is atrophy of the lentiform nuclei with abnormal low signal intensity in the posterior putamen (long arrow) and in the lefi thalamus (short arrow) $(A)$. These regions are high signal intensity $(B)$. There is a deficit in myelination posteriorly $(A)$. Abnormal increased SI around the lentiform nuclei and insula cortex is best seen in (C) 


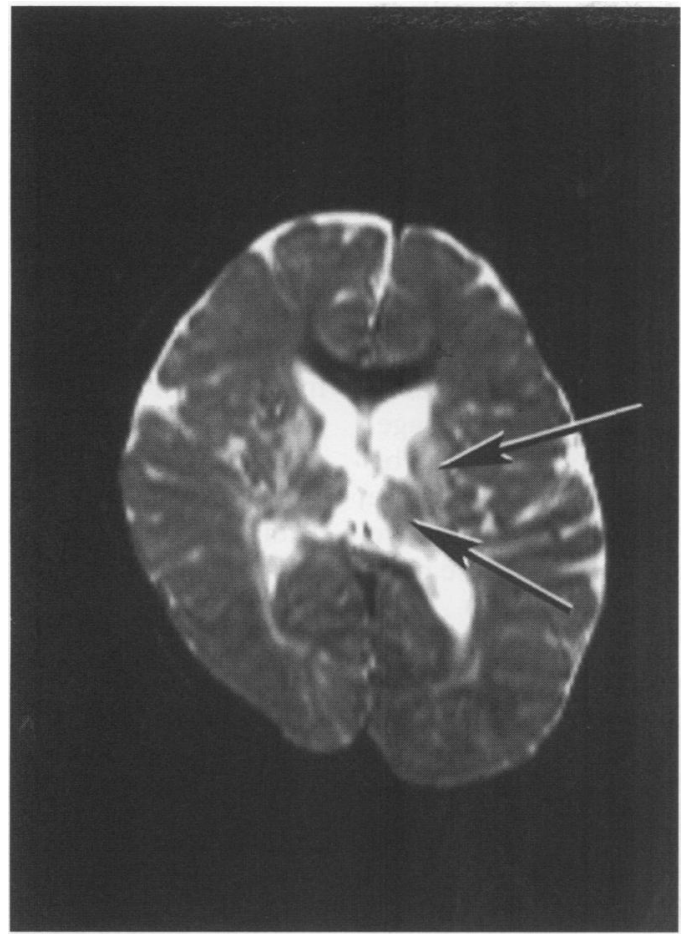

Figure 6 Girl with grade II HIE, aged 15 months (case 15). $T_{2}$ weighted spin echo sequence $S E 3000 / 120$. There is pronounced bilateral atrophy of the lentiform nuclei, thalami, and head of the caudate nucleus with abnormally high and low signal intensities (arrows).

white matter atrophy with increased $T_{2}$ in the centrum semiovale and periventricular white matter. Decreased $T_{2}$ was seen in the cortex adjacent to the interhemispheric fissure (NMR score 9). The basal ganglia were of normal appearance. Her neonatal scan showed brain swelling and widespread cortical highlighting.

Severe developmental delay $(n=5)$ :

Five infants had a central motor deficit, severe developmental delay, and secondary microcephaly. (The optimality scores ranged from 8.1 -14.6.) All had secondary microcephaly.

Three infants had a mixed quadriplegia with athetoid or dystonic movements of the upper limbs, hypotonia of the trunk, and increased tone in the lower limbs (cases 12,13,14). Two of these infants had no hand function, but the third who had milder dystonic posturing was able to transfer objects at 17 months of age (case 12). This infant had no abnormal facial appearances and showed good vocalisation with a vocabulary of two words.

A fourth infant had a generalised increase in tone with dystonic posturing of the right hand (case 10). She was able to sit independently and spoke a few words. The sixth infant with a central motor deficit and severe delay had generally decreased tone with severe visual problems. He was able to sit with minimal support and was able to transfer objects but had no fine hand manipulation at 18 months (case 11).

A follow up MRI scan showed severe basal ganglia changes with atrophy and cyst formation in the three infants with severe dystonic and athetoid movements. They also showed a loss of white matter and an abnormal increase in SI around the primary corticospinal tracts on $T_{2}$ WSE scans (NMR scores $\left.16,21,31\right)$. One infant had a posterior parietal infarct. These infants had abnormal SI in the basal ganglia and thalami, the cortex, and in the subcortical and periventricular white matter on neonatal scans.

Two further infants (cases 10,11) with severe developmental delay showed a different pattern of abnormalities on MRI.

Follow up MRI showed- loss of tissue secondary to infarction in both infants. The basal ganglia were affected in both infants, although these were asymmetrical in case 10 . The images did not show any substantial increase in SI on the $T_{2}$ WSE scan. Myelination was decreased in both infants but this decrease was extreme in case 11 (NMR scores 20 and 28).

Their neonatal scans showed multiple areas of infarction involving the white matter and cortex and abnormal SI within the basal ganglia and thalami.

Very severe developmental delay $(n=2)$ :

Two infants made no discernible development. (Optimality scores were 7.6 and 6.3.) Both had secondary microcephaly, severe extensor posturing, ongoing convulsions, persistent feeding problems necessitating nasogastric or gastrotomy feeding.

On follow up MRI both infants had extensive atrophy of the basal ganglia, a decrease in white matter, and a substantial decrease in myelination. On the $T_{2}$ WSE sequence images there was an increase in SI along the primary corticospinal tracts (fig 3C) (NMR scores 39 and 40 ). Their neonatal scans showed abnormal SI throughout the basal ganglia and thalami with highlighting of the cortex, which was most severe around the central fissure and interhemispheric fissure.

Table 4 shows the details of the follow up optimality score with the neonatal and follow up TBG and total NMR scores. There was a strong positive correlation between the optimality score and the neonatal TBG $(P=0.0004)$, the neonatal NMR $(P=0.0034)$, the follow up TBG $(P=0.0002)$ and the follow up NMR score $(P=0.0012)$.

\section{Discussion}

In neonates with HIE early MRI may show evidence of brain swelling. This clears during the first week of life as signs of permanent damage become more obvious. Abnormal signal intensities may be seen in the basal ganglia and thalami, the internal capsule, the periventricular and subcortical white matter and in the cortex. ${ }^{10}$ We have shown that in term infants with HIE there may be late MRI abnormalities within the basal ganglia, the cortex, the white matter, the hippocampi, the insulae and the brainstem. There were no normal follow up scans and infants with a normal outcome showed patchy abnormal SI within the periventricular white matter, which was most noticeable posteriorly. Persistent basal ganglia abnormalities were always associated with an abnormal outcome, the severity and character of which were related to the degree of abnormality seen. Infants with extensive white matter abnormalities all had an abnormal outcome, but those without pronounced basal ganglia abnormalities had less severe problems. There was a strong correlation between the 
neonatal and late NMR and TBG scores and the outcome of the child, as measured by the optimality score.

Infants with a normal outcome all showed patchy white matter changes on their follow up scans. These changes were only detectable on $\mathrm{T}_{2}$ weighted images and were difficult to distinguish from unmyelinated white matter before 1 year of age. It is not possible to say whether these changes will persist with time or whether they will be associated with more minor neurological dysfunction at school age. Mild or grade I HIE is always considered to be a benign disorder, but to our knowledge there have been no published studies that have looked at the fine motor function in these children at 5 years plus.

Byrne documented the development of cerebral palsy in three children with normal scans at 3 months of age, but abnormal scans at 8 months of age, with the development of delayed myelination and cortical atrophy. ${ }^{3}$ We did not see this type of deterioration in any of our infants. Deterioration of a previously normal scan may imply that subtle but clinically important changes - that is, in the cortex or white matter - may have been difficult to identify early on. This could be because the changes were time dependent or because the imaging sequences used were not optimal. We have shown that infants with patchy periventricular white matter changed on $T_{2}$ WSE scans at 1 year of age may have only had signs of brain swelling on neonatal $T_{1}$ WSE sequence scans. ${ }^{10}$ The signs of swelling were only visible for a few days. Alternatively, a child with an abnormal outcome but a normal early scan may have an additional diagnosis, such as a metabolic problem, giving rise to ongoing damage within the brain. Isolated delay in myelination may be one of the earliest MRI changes in children with a metabolic disorder. ${ }^{14}$ We have seen isolated deficit in myelination in infants with neurological problems not due to HIE, although they may have had fetal distress and abnormal deliveries in addition to their primary problem. In this study a deficit in myelination was only seen in association with other structural changes within the brain.

The basal ganglia are a common site for abnormalities in the neonatal period, but only the more severe and persistent lesions seem to be associated with a central motor deficit. Atrophy or cysts in the basal ganglia have been reported in retrospective studies of children with cerebral palsy and are felt to be good evidence in support of the occurrence of significant perinatal injury, as opposed to preexisting prenatal abnormalities. ${ }^{1516}$ The type of motor deficit may depend on the exact site and extent of basal ganglia injury and whether there is extension into the midbrain and damage in other parts of the brain. We have shown that severe basal ganglia injury is associated with severe spastic quadriplegia with pronounced feeding difficulties and ongoing convulsions. The feeding difficulties are probably related to involvement of the midbrain which is more difficult to assess on MRI but is associated with severe basal ganglia injury on necropsy specimens. ${ }^{17}$ In this study generalised atrophy with or without cyst formation in the basal ganglia was also associated with abnormal SI within the hippocampus, a pattern of injury that has been attributed to a period of total asphyxia using animal models. ${ }^{18}$

Less severe damage with atrophy and cyst formation within the lentiform nuclei was seen in three infants, all of whom had a mixed quadriplegia with athetoid and or dystonic movements. A further infant with asymmetrical basal ganglia abnormalities had dystonic movements in the contralateral arm only. Localised cysts in the posterior part of the putamen without generalised atrophy are strongly associated with the development of athetoid quadriplegia. ${ }^{19}{ }^{20}$ Interestingly, all three infants in this study with a mixed quadriplegia with athetoid movements had microcephaly and severe early feeding problems, with no suck and poor swallow. This contrasts to our previous findings in three infants with a purer athetoid quadriplegia all of whom maintained a normal head circumference and all of whom were bottle feeding by 2 weeks of age. ${ }^{19}$

White matter atrophy is a common finding on the follow up scans of infants with HIE. Areas of white matter atrophy were distinguished because of the lack of myelin and the collapsing down of cortical gyri on IR sequences, but it was not possible to differentiate atrophy of the subcortical white matter from the periventicular white matter using the IR sequence alone. Abnormally increased $T_{2}$ probably representing secondary gliosis was, however, easily seen on the $T_{2}$ WSE sequences and permitted a more accurate localisation of white matter damage. A delay or deficiency in myelination was easiest to identify on IR sequence scans, which was also the finding in Byrne's study. Although NMR is the imaging method of choice for assessing myelination, the patterns seen only grossly approximate the complex process of central nervous system development.

Three changes on neonatal scans were associated with the subsequent appearance of white matter atrophy, areas of infarction, a streaky appearance in the white matter of the centrum semiovale, and subcortical low signal on IR sequence. Infants with a streaky appearance in the white matter during the first week of life did not develop cysts and the aetiology of the eventual white matter atrophy remains obscure. Low signal in the subcortical white matter was associated with cortical highlighting. The cortical highlighting appeared in the first week of life but the adjacent areas of white matter low signal did not appear for at least another seven days. ${ }^{10}$ The timing of the subcortical changes reflects either a primary ischaemic process in the tissue with cyst formation appearing 10 to 14 days later, or alternatively, it may be secondary to the damage within the cortex. The cortex and subcortical white matter around the central fissure in the region of the primary corticospinal tracts were most frequently involved and at follow up detected as increased $T_{2}$. This region may be more sensitive to asphyxial damage at term when it is actively myelinating. ${ }^{2}$ 
The clinical relevance of the cortical highlighting seen on neonatal IR scans is uncertain. The SI changes observed are in keeping with a haemorrhagic lesion. The MRI appearance of cortical highlighting may correspond to the increased cortical echogenicity identified using cranial ultrasonography in infants with HIE. This increased echogenicity corresponded to areas of cortical laminar necrosis on histological examination. ${ }^{21}$

On follow up MRI scans it was possible to identify persistent low signal on $T_{2}$ WSE sequence within the cortex along the interhemispheric fissure. This would be consistent with old haemorrhage. Cortical laminar abnormalities, giving abnormal SI on $\mathrm{T}_{2}$ weighted images have been described by other workers on late MRI scans in children with a history of perinatal hypoxic-ischaemic brain injury and spastic quadripareses. ${ }^{22}$

Only one of our infants had pronounced cortical and subcortical white matter changes with relatively preserved basal ganglia and she has done remarkably well (case 9). The clinical importance of early and late cortical and subcortical changes following HIE are poorly understood and further insight may be gained with more sophisticated imaging combined with specific electrophysiological studies in children with no evidence of basal ganglia abnormalities.

The dentate nuclei of the cerebellum are sensitive to hypoxic-ischaemic damage in animal models but there are few reports of cerebellar abnormalities following hypoxicischaemic injury in neonates. ${ }^{23}{ }^{24}$ The cerebellum may be damaged along with the thalami and hippocampus as part of the injury following severe total asphyxia. In Eken's study of infants with HIE, histological cerebellar lesions were seen in 11 of 20 infants, ${ }^{21}$ but it is not clear what pattern of injury these infants had sustained.

As all infants in our study survived, the insults they sustained may have been insufficient to cause major lesions within the cerebellum. The only abnormality seen in this study was a deficit in myelination within the cerebellum, and this was associated with a generalised deficit of myelin throughout the brain. Although mild degrees of atrophy may have been present but difficult to identify, the cerebellum seems to remain intact when the rest of the brain is grossly damaged.

MRI permits the detection of exact patterns of early and late damage in infants with HIE. Some correlations with different patterns of damage can be made. In most cases, however, we are still not able to correlate accurately specific areas of damage with specific clinical sequelae. This is partly because in infants with HIE few areas are damaged in isolation. The imaging techniques used did not measure function and while severe degrees of atrophy are identifiable, these could not be measured and less severe atrophy may well have gone undetected. Further studies using a combination of electrophysiological and MRI techniques will allow us to answer some of the questions on the functional relevance of specific abnormalities.
Registration and subtraction of serial volumetrically acquired images should help in measuring and detecting even mild degrees of atrophy, and in detecting milder deficiencies and abnormalities in the progression and patterns of myelination. ${ }^{25}$

Mary Rutherford was supported by Scope (formerly the Spastics Society). We are grateful to Professor Graeme Bydder for his guidance and support.

1 McCardle CB, Richardson CJ, Hayden CK, Nicholas DA, Amparo EG. Abnormalities of the neonatal brain: MR imaging. II. Hypoxic-ischaemic brain injury. Radiology 1987: 163:395-403.

2 Barkovich AJ, Truwit LT. Brain damage from perinatal asphyxia: Correlation of MR findings with gestational age. Am $\mathcal{F}$ Neuroradiol 1990;11: 1087-96.

3 Byrne PR, Welch MA, Johnson J, Darrah M, Piper M. Serial magnetic resonance imaging in neonatal hypoxicischaemic encephalopathy. $\mathcal{F}$ Pediatr $1990 ; 117 ; 694-700$.

4 Keenay SE, Adcock EW, McArdle CB. Prospective observations of 100 high-risk neonates by high-field ( 1.5 Tesla) magnetic resonance imaging of the central nervous magnetic resonance imaging of the central nervous encephalopathy. Pediatrics 1991; 87: 431-8.

5 Roland EH, Hill A.MR and CT evaluation of profound neonatal and infantile asphyxia. Am $\mathcal{F}$ Neuroradiol 1992;13: 973-5.

6 Baenziger OE, Martin M, Steinlin M, Good R, Largo R, Burger S, et al. Boltshauser: Early pattern recognition insevere perinatal asphyxia: a prospective MRI study. Neuroradiology 1993; 37: 437-42.

7 Kuenzle C, Baenziger O, Martin E, Thun-Hohenstein L, Steinlin M, Good M, et al. Prognostic value of early MR imaging in term infants with severe perinatal asphyxia. Neuropaediatrics 1994;4:191-200.

8 Rutherford MA, Pennock JM, Dubowitz LMS. Hypoxicischaemic encephalopathy: A comparison of cranial ultrasound and magnetic resonance imaging with outcome. Dev Med Child Neurol 1994;36:813-25.

9 Martin E, Barkovich AJ. Magnetic resonance imaging in perinatal asphyxia. Arch Dis Child 1995;72: F62-70.

10 Rutherford MA, Pennock JM, Schwieso JE, Cowan FM, Dubowitz LMS. Hypoxic-ischaemic encephalopathy: Early magnetic resonance imaging findings and their evolution. Neuropaediatrics 1995; 26: 183-91.

11 Sarnat HB, Sarnat MS. Neonatal encephalopathy following fetal distress: a clinical and electrophysiological study. Arch Neurol 1976; 33: 696-705.

12 Hajnal JV, de Coene B, Lewis PD, Baudauin CJ, Cowan FM, Young IR, et al. High signal regions in normal white matter shown by heavily T2 weighted CSF nulled inversion recovery sequences. f Comput Assist Tomogr 1992;16:506-13.

13 Dubowitz L, Dubowitz V. The neurological assessment of the preterm and full term newborn infant. Clinics in Developmental Medicine. No 79. London:SIMP: 1981.

14 Ball WS. Magnetic resonance imaging of the infant brain Semin Ultrasound 1991;12:379-409.

15 Barkovich AJ. MR and CT evaluation of profound neonatal and infantile asphyxia. Am $\mathcal{F}$ Neuroradiol 1992; 13: 959-72.

16 Truwit CL, Barkovich AJ, Koch TK, Ferriero DM. Cerebral palsy: MR findings in 40 patients. Am $\mathcal{F}$ Neuroradiol 1992;13:67-78.

17 Roland EH, Hill A, Norman MG, Flodmark O, MacNab AJ. Selective brainstem injury in an asphyxiated newborn. Ann Neurol 1988;23:89-92.

18 Myers RE. Two patterns of brain damage and their conditions of occurrence. Am $\mathcal{f}$ Obstet Gynaecol 1972;112:246-76.

19 Rutherford MA, Pennock JM, Murdoch-Eaton DM, Cowan FM, Dubowitz LMS. Athetoid cerebral palsy and cysts in the putamen after hypoxic-ischaemic encephalopathy. Arch Dis Child 1992; 67: 846-50.

20 Yokochi K, Aiba K, Kodama M, Fujimoto S. Magnetic resonance imaging in athetotic cerebral palsied children. Acta Paediatr Scand 1991;80:818-23.

21 Eken P, Jansen GH, Groenendaal F, Rademaker KJ, de Vries S. Intracranial lesions in the fullterm infant with hypoxicischaemic encephalopathy. Neuropaediatrics 1994; 25: 301-7.

22 Van der Knaap MS, Smit LS, Nauta JP, Lafeber HN, Valk J. Cortical laminar abnormalities - Occurrence and clinical significance. Neuropediatrics 1993; 24: 143-8.

23 Dambska M, Dydyk L, Szretter T, Wozniewicz J, Myers RE. Topography of lesions in newborn and infant brains following cardiac arrest and resuscitation. Biol Neonate 1976;29:194-206.

24 Pasternak JF, Predey TA,Mikhael MA. Neonatal asphyxia: Vulnerability of basal ganglia, thalamus and brainstem. Pediatr Neurol 1991;7:147-9.

25 Hajnal JV, Saeed N, Oatridge A Williams EJ, Young IR, Bydder GM. Detection of subtle brain changes using subvoxel registration and subtraction of serial MR images. $\mathcal{f}$ Comp Assist Tomogr 1995;19:677-91. 